\title{
LA IMPORTANCIA DE LA PARTICIPACION COMUNITARIA EN LOS PLANES DE MANEJO DE FAUNA SILVESTRE EN EL NOR ORIENTE DEL PERU
}

Pablo Puertas ${ }^{1}$, Richard Bodmer ${ }^{2}$, José López Parodi $^{1}$, Javier del Aguila $^{1}$ y Arsenio Calle ${ }^{1}$

\section{RESUMEN}

En este documento se analizan las acciones de manejo de fauna silvestre con participación comunitaria realizadas tanto en la Reserva Comunal Tamshiyacu - Tahuayo (RCTT) como en la Reserva Nacional Pacaya Samiria (RNPS), nor-oriente del Perú. El objetivo fue determinar métodos para obtener y analizar información biológica con participación comunitaria. Las áreas de estudio comprendieron: 1) el área del Tahuayo-Blanco, en la RCTT, 2) el área del canal de Puinahua, en la RNPS y 3) el área del Samiria-Marañón, en la RNPS. La información fue obtenida con ayuda de cazadores locales, denominados localmente como inspectores, en el caso de la RCTT y como expertos, en el caso de la RNPS. Esta información incluyó número de cazadores, especies cazadas, tiempo de captura, campamentos utilizados, tiempo de residencia y accesibilidad a los sitios de caza. Consideramos una necesidad realizar investigaciones para mejorar el sistema de co-manejo; asimismo, la metodología descrita en este estudio puede ser utilizada también para la pesca y forestería. Una recomendación es incorporar el análisis de fauna silvestre realizado por las comunidades locales. Adicionalmente, el manejo debe tomar en consideración la zonificación de las áreas de caza, rotación de la caza y una reducción de las cosechas no sostenibles. El manejo de fauna silvestre con participación comunitaria requiere de consideraciones políticas que involucren acciones tendientes al mejoramiento del hábitat y al empoderamiento de las comunidades locales en las áreas de co-manejo.

Palabras clave: Participación comunal, manejo de fauna silvestre, co-manejo comunal, Reserva Comunal Tamshiyacu-Tahuayo, Reserva Nacional Pacaya Samiria.

1 Programa Integral de Desarrollo y Conservación Pacaya Samiria. Junglevagt for Amazonas (WWF, AIF/DK). Pevas 120, Iquitos (Perú). E-mail: ppsgrupo@ rail.org.pe

2 University of Kent at Canterbury. Canterbury, Kent CT2 7NS, England. E-mail: R. Bodmer@ukc.ac.uk 


\begin{abstract}
This study analyses the community based co-management of wildlife in the Reserva Comunal Tamshiyacu-Tahuayo (RCTT) and the Reserva Nacional Pacaya Samiria (RNPS), northeastern Peru. The objective of the research was to determine methods to obtain and analyze biological information with community participation. The study comprised : 1) the Tahuayo-Blanco area in the RCTT, 2) the Canal de Puinahua area in the RNPS, and 3) the Samiria- Marañon area in the RNPS. The information was collected with the help of local hunters called hunting inspectors in the case of the RCTT, and hunting experts in the case of the RNPS. This information included the number of hunters, time out hunting, species hunted, camps, time of residence, and accessibility to the hunting sites. Further research needs to focus on the improvement of the co-management system in order to comply with other activities such as fishing and forestry. One recommendation would be to incorporate wildlife analysis by the local communities. In addition, the current management needs to take into consideration the zoning of hunting areas, rotation of hunting, and reduction of unsustainable harvests. Community-based wildlife management requires political considerations that involve actions toward the enrichment of the habitat and the empowering of the local communities in the co-management areas.
\end{abstract}

Key words: Community participation, wildlife management, community based-comanagement, Reserva Comunal Tamshiyacu-Tahuayo, Reserva Nacional Pacaya Samiria.

\title{
1. INTRODUCCION
}

La extracción de recursos naturales es una de las principales actividades de los habitantes amazónicos, sea de los que viven en los bosques inundables que los que viven en tierra firme (Beckerman, 1994; Bodmer, 1994). Una de las áreas de tierra firme es el Tahuayo-Blanco y, en el caso de la Reserva Nacional Pacaya Samiria, éstas comprenden las siguientes zonas: 1) canal del Puinahua, en la cuenca del Ucayali y 2) zona del Marañón-Samiria, ambas comprendidas en las áreas de influencia del Programa Integral de Desarrollo y Conservación Pacaya Samiria (PPS). La gente local de esas áreas, como de otros bosques tropicales, utilizan en la alimentación, preferentemente, animales de tamaño pequeño, mientras que los de tamaño mediano a grande, contribuyen a su economía a través de la venta de carne de monte (Redford y Robinson, 1991; Bodmer, 1994; Bodmer et al., 1997). 
La fauna silvestre juega un papel importante en la vida de la gente amazónica ya que es utilizada para la obtención de ornamentos, pieles, plumas, mascotas, animales domesticados, zoológicos; para uso biomédico, caza deportiva, turismo, materiales de construcción y en la percepción de su mundo cultural (Aspelin, 1975; Flowers, 1983, 1994; Yost y Kelley, 1983; Vickers, 1991; Redford y Robinson, 1991; Dufour, 1994). En consecuencia, la fauna silvestre en los bosques tropicales puede ser fácilmente sobre-explotada y reducida a la extinción causando cambios en la dinámica poblacional de las especies e influyendo en el desenvolvimiento de las comunidades humanas (Robinson y Redford, 1994; Robinson y Bodmer, 1999).

Debido a que la mayoría de los habitantes locales explotan los recursos de caza de modo no sostenible, algunas comunidades han empezado a asumir responsabilidades para conservar sus recursos, ya sea por iniciativa comunitaria propia o representadas por grupos comunales organizados. Estudios conducidos tanto en la Reserva Comunal Tamshiyacu-Tahuayo (RCTT) como en la Reserva Nacional Pacaya Samiria (RNPS), indican que la gente local ha empezado a manejar la fauna silvestre como una alternativa de conservación (Bodmer y Puertas, 2000). Sin embargo, la implementación del manejo comunal de fauna silvestre requiere contar con datos de campo que concuerden con la realidad de la gente local.

Consideramos que las sugerencias proporcionadas en este documento podrían ayudar a las comunidades locales y a los planificadores de áreas naturales protegidas a establecer un sistema de caza sostenible a través de la implementación de políticas de manejo comunal, fundamentadas con información biológica relevante.

\section{ANTECEDENTES SOBRE ACCIONES DE MANEJO COMUNAL DE FAUNA SILVESTRE}

Los antecedentes de manejo de recursos naturales en Sudamérica indican que, mayormente, se han tomado medidas proteccionistas para salvaguardar áreas con especies de fauna y flora amenazadas (Townsend, 1996). Sin embargo tales medidas, debido a sus visión centralista, legalista y burocrática se dieron y se siguen dando sin la participación de las comunidades siendo, por consiguiente, deficiente en su funcionalidad y aplicación práctica. Este es el caso de los programas de manejo de recursos naturales en el nor-oriente peruano. Por ejemplo, el caso de la Reserva Nacional Pacaya-Samiria, que fue creada en 1982 sin tomar en consideración a los habitantes del lugar que ancestralmente han ocupado esas tierras. 
Muchos de los habitantes de las áreas protegidas del nor-oriente peruano son gente pobre que usa los recursos de las áreas naturales protegidas para fines de subsistencia y comercialización, principalmente en los mercados de las ciudades y pueblos de Iquitos, Requena, Pucallpa, Nauta y Yurimaguas. Entre esos bosques, la RCTT y la RNPS aprovisionan de carne de monte a los pobladores. Sin embargo, en el caso de los bosques de la RNPS, éstos se encuentran entre los más amenazados de la Amazonía debido a su fácil accesibilidad para la extracción de los recursos naturales por vía fluvial (Bodmer et al., 1999).

La conservación de los recursos naturales en la Reserva Nacional Pacaya Samiria, a través del sistema de guardaparques, no viene dando los resultados esperados debido a que los lugareños no están involucrados en el proceso de la toma de decisiones con el INRENA. Esto ha dado lugar a una triste historia de acciones represivas, abuso, subestimación y desprecio hacia la gente local la cual, desde hace tiempo, está en contra del sistema de guardaparques. Sin embargo, recientemente, las comunidades locales se sienten motivadas debido a la iniciativa del gobierno peruano de hacer participar, por primera vez, a las poblaciones locales, en la toma de decisiones para la elaboración del Nuevo Plan Maestro de la RNPS; es decir, participarán haciendo llegar al equipo planificador de la RNPS sus propuestas de zonificación, las diversas formas de uso de recursos naturales para su posterior aprovechamiento sostenido, bajo planes comunales de manejo.

Las experiencias de conservación en aquellas regiones con bosques tropicales, tales como Brasil, Bolivia y Africa, indican que una de las mejores maneras para conservar los recursos naturales es involucrando a las comunidades locales (Metcalfe, 1994; Townsend, 1996). Por ejemplo, el éxito de la reserva Mamirauá, en la Amazonía del Brasil, se debe a las iniciativas de manejo de recursos naturales con participación comunitaria.

\subsection{Sistemas de uso sostenible de la fauna silvestre mediante el manejo comunal}

El manejo comunal apunta a que las comunidades rurales jueguen un papel fundamental en la conservación de las especies y ecosistemas, así como en el manejo mediante el uso sostenible de los bosques (Little, 1994). Las comunidades locales que viven en los estuarios costeros y en los bosques tropicales y de savanas empezaron a tomar responsabilidades para manejar y conservar los recursos naturales (Maltby et al., 1992; Bodmer, 1994; Poffenberger, 1994). 
El manejo comunal de fauna silvestre funciona mejor si es co-manejado con investigadores, Organismos No gubernamentales (ONG) y autoridades de gobierno (Pinkerton, 1989). El impacto real del manejo comunal puede ser minuciosamente analizado únicamente mediante estudios de largo plazo. Estos permiten un mejor entendimiento de: 1) las iniciativas y acciones de manejo comunal basadas en el comanejo y 2) las limitaciones de los recursos biológicos y las respuestas al manejo. El manejo comunal de la RCTT comenzó mucho antes del establecimiento legal de la reserva en 1991. Acciones medioambientales tomadas por las comunidades del alto Tahuayo tuvieron una mayor influencia para crear la reserva. Las comunidades se dieron cuenta de la degradación de los recursos naturales de sus bosques; esto permitió que tomaran iniciativas comunales para proteger los recursos naturales (Bodmer, 1994).

Las comunidades del alto Tahuayo implementaron una serie de reglas para la extracción de recursos naturales y el uso de la tierra que se determinan, por consenso, en cada comunidad. Las comunidades también tomaron acuerdos formales relacionados a reglas de acceso y puestos de vigilancia. Todavía no están precisadas, entre las comunidades y los promotores, las mejores técnicas de manejo y es por eso que es necesario desarrollar una fuerte ligazón entre las investigaciones biológicas y los promotores o trabajadores de extensión (Bodmer y Puertas, 2000).

La caza de animales es una de las principales actividades que se están realizando en la zona de subsistencia de la RCTT. El primer plan de manejo de fauna silvestre aplicado por las comunidades fue con respaldo gubernamental y se centró en un tipo de caza. En el Tahuayo, durante la década de 1980, madereros foráneos extrajeron más del 50\% de mamíferos de caza que los habitantes locales (Bodmer et al., 1988). Para el desarrollo de esta actividad, los patrones habilitaron a sus trabajadores con escopetas y cartuchos en vez de proporcionarles alimentos básicos, razón por la cual los madereros cazaron en exceso muchas especies de fauna silvestre. Las comunidades expresaron su preocupación a las autoridades de gobierno, quienes a su vez promovieron el área como "una reserva en estudio". Esta categorización legal hizo posible terminar con las concesiones madereras en 1988 y reducir la sobre-explotación de fauna silvestre por los madereros. Como consecuencia de esto, en 1991 las comunidades observaron que muchos cazadores comerciales habían comenzado a ingresar al área. Con la ayuda de los promotores, las comunidades del alto Tahuayo empezaron a implementar el sistema de vigilancia, prohibiendo a los cazadores de la ciudad la entrada al área. En cambio, se permitió el ingreso de la gente local a la zona de subsistencia. 
El establecimiento de planes de manejo con participación comunal requiere integrar información biológica de las especies de caza, economía y uso sostenible con los deseos de las comunidades locales. Por consiguiente, se requiere involucrar a todos los actores interesados como son: 1) las comunidades locales, 2) los investigadores, 3) los promotores de las ONG y 4) los representantes de instituciones gubernamentales.

\subsection{Estudios de largo plazo de las poblaciones de fauna silvestre: ¿Puede el manejo comunal conservar las poblaciones de fauna silvestre?}

Estudios de largo plazo conducidos sobre las sacas y las poblaciones de mamíferos, en la Reserva Comunal Tamshiyacu, examinaron el impacto del manejo comunal. Este tipo de estudio se viene haciendo también en otras áreas de la Amazonía Peruana, como la Reserva Nacional Pacaya Samiria. Programas y planes de manejo fueron establecidos en la RCTT con participación comunal en el manejo de fauna silvestre mediante actividades de investigación y extensión comunal. Esos planes de manejo desarrollaron prácticas que restringieron el acceso a la caza, capacitaron a registradores de caza y pesca, implementaron un sistema de cuotas e impuestos, así como el desarrollo de un programa de cosecha orientado a la caza de animales machos (ACA 1998).

En el caso de la RCTT, los estudios sobre las poblaciones de animales incluyeron análisis de densidades, análisis de estructura de edades, modelos de cosechas y de sostenibilidad. De acuerdo a los análisis efectuados, resultó que los primates y el tapir o sachavaca eran sobrecazados, en cambio, los artiodáctilos y roedores grandes, aparentemente, no eran sobrecazados (Bodmer, 1994; 1995; Bodmer et al., 1999). De este modo, la información obtenida permitió a los promotores transmitir adecuadamente a las comunidades locales la necesidad de reducir la caza de primates y de la sachavaca, así como también la de mantener a niveles normales la cosecha de artiodáctilos y de roedores grandes.

Un análisis preliminar indica que, luego de cuatro años de manejo comunal en la RCTT, la cosecha de artiodáctilos mostró una ligera, pero no significativa, diferencia entre 1991, 1994 y 1995 medidos como el número de animales cosechados $/ 100 \mathrm{~km}^{2}$ por año. Similarmente, la cosecha de los roedores grandes y de la sachavaca no mostró diferencias significativas entre 1991, 1994 y 1995. Sin embargo, los cazadores cosecharon significativamente menos primates entre 1991 y 1995 lo que, al parecer, fue debido a acciones de manejo comunal (ACA 1998). 
En el caso de la RNPS, la inundación de los ríos Marañón y Samiria influencian la abundancia de animales. Es por eso que la caza ocurre mayormente en la creciente, entre marzo y junio, y con menor intensidad en la vaciante, entre julio y noviembre. La caza es una actividad importante para la gente que vive en la zona de amortiguamiento de la RNPS, razón por la cual, estudios sobre la dinámica poblacional de las especies de caza fueron conducidos en la zona del Samiria-Marañón (Bodmer et al., 1999). Entre los resultados, se reporta que muchas de las especies están siendo sobrecazadas y que urge una reducción a niveles sostenibles para evitar sus exterminación.

Por consiguiente, en el marco del Proyecto Manejo y Conservación Participativa de Recursos Naturales del PPS se desarrolla un plan de manejo de fauna silvestre con participación comunitaria teniéndose en consideración ciertas características específicas, como modo de vida del animal, tipo de alimentación, época de pariciones, entre otros aspectos ecológicos y de la dinámica poblacional. Como complemento a las acciones de manejo comunal, también se proyecta desarrollar un programa de educación ambiental. Para ello, se considera importante continuar con los estudios de fauna si se desea realizar un programa de manejo a largo plazo con participación activa de las comunidades locales, así como de instituciones gubernamentales y no gubernamentales como el Instituto Nacional de Recursos Naturales (INRENA), Ministerio de Agricultura, Instituto de Investigaciones de la Amazonía Peruana (IIAP), Universidad Nacional de la Amazonía Peruana, Programa Integral de Desarrollo y Conservación Pacaya Samiria, World Wildlife Fund-Oficina Programa Perú, Pro Naturaleza, entre otras instituciones gubernamentales y no gubernamentales. Ello, en un proceso de co-gestión o gestión conjunta para dar viabilidad al manejo comunal de fauna silvestre.

\subsection{Conservación de hábitats de la fauna silvestre}

El manejo de hábitats, tanto en la Reserva Nacional Pacaya Samiria como en la Reserva Comunal Tamshiyacu-Tahuayo, incluye programas agroforestales diseñados para beneficiar tanto a las comunidades locales como a la fauna silvestre. Las palmeras son frecuentemente taladas en ambas reservas para obtener los frutos. Los animales de caza también se alimentan de los frutos de palmeras. La reducción del área de palmeras incide negativamente en la capacidad de carga de animales de caza y por consiguiente reduce el número de animales que pueden ser cazados sosteniblemente (Bodmer et al., 1999). La sobre-explotación de la palmera de aguaje (Mauritia flexuosa) preocupa a mucha gente, principalmente del Samiria, Marañón y alto Tahuayo, debido a consecuencias económicas por la pérdida de los frutos y de la 
poblaciones de fauna silvestre. En el caso de la RCTT, desde 1992 más de 20 familias del alto Tahuayo plantaron y manejaron grandes cantidades de palmeras de aguaje. La recuperación de hábitats en dicha reserva puede ahora ser posible si más familias continúan plantando y manejando palmeras de aguaje (Penn, 1994, 1999; ACA, 1998).

\section{PERIODO Y AREAS DE ESTUDIO}

Las acciones realizadas en el marco del presente estudio comprendieron los años de 1992 a 1996 en la Reserva Comunal Tamshiyacu-Tahuayo y los años de 1994 a 2000 en la Reserva Nacional Pacaya Samiria.

\subsection{El área de estudio en la Reserva Comunal Tamshiyacu-Tahuayo (RCTT)}

El área de estudio está localizada en la parte media y superior del río Blanco, afluente del río Tahuayo y comprende la zona de establecimientos humanos y la de subsistencia de la RCTT (Fig. 1). La RCTT está localizada en el nor-oriente peruano y cubre un bosque continuo de 322500 ha, conformado, predominantemente, por bosques de altura $(>75 \%) \mathrm{y}$, en menor proporción, por bosques inundables (Bodmer et al., 1997).

La mayoría de los habitantes rurales de la reserva son gente detribalizada, conocida como "ribereños" (Puertas y Bodmer, 1993; Bodmer et al., 1997). Ellos, comúnmente, practican la pesca, la agricultura, la caza, la extracción a pequeña escala de productos maderables y la colección de otros productos del bosque como frutos, nueces y fibras (Coomes, 1992).

\subsection{El área de estudio en la Reserva Nacional Pacaya Samiria (RNPS)}

El área de estudio en la RNPS comprende las zonas de influencia del Programa Integral de Desarrollo y Conservación Pacaya Samiria y de las comunidades indígenas organizadas de AIDECOS (Asociación Indígena de Desarrollo y Conservación del Samiria) y ADECOP (Asociación de Desarrollo y Conservación del Puinahua). Una zona se encuentra situada en el curso medio del canal del Puinahua y la otra se ubica en el curso inferior de los ríos Samiria y Marañón (Fig. 1). 
La RNPS es la única área protegida de la Amazonía Peruana que está formada, principalmente, por hábitats inundables. Sin embargo, esos hábitats se encuentran entre los más vulnerables de la Amazonía por la calidad del suelo y la accesibilidad a la extracción de madera, caza y pesca (Bodmer et al., 1999).

A la población, en la RNPS, también se le denomina localmente ribereños y está constituida, en un $80 \%$, por indígenas pertenecientes al grupo étnico Cocama y cerca de un $20 \%$ por otros grupos indígenas y por mestizos y blancos (PPS 1995). Entre las principales actividades destacan la pesca seguida por la agricultura de arroz, maíz, frijol, maní, plátano y yuca, así como la caza de animales silvestres y la recolección de productos del bosque, como aguaje y chonta.

\section{METODOS}

En este estudio, destacamos el método participativo utilizado con las comunidades locales de la RCTT y su extensión en la RNPS para la elaboración y el desarrollo de planes de manejo de fauna silvestre.

\subsection{La metodología participativa para el manejo de fauna silvestre}

El método participativo se basa en generar interés en las comunidades locales por el manejo de fauna silvestre, involucrando a investigadores y extensionistas o promotores, para trabajar con los cazadores, cuando se evalúa el impacto de las cosechas (Bodmer y Puertas, 2000). Uno de esos métodos es el registro de la caza, que involucra a la familia de los cazadores en la colección de datos. Este método participativo ayuda a los investigadores, promotores y cazadores a encontrar caminos comunes para discutir asuntos de fauna silvestre.

La información sobre las especies de caza y la variación de la extracción, según las temporadas del año, fueron usadas para interpretar la dinámica de la caza y evaluar su impacto sobre las poblaciones de caza tanto dentro como fuera de la reserva, en un sistema de fuente-sumidero. Es decir, para observar si los animales de las zonas con poca caza (zonas fuente) están repoblando las zonas con caza persistente (sumideros) o viceversa.

La comunidad 7 de Julio, situada en el río Blanco, fue el punto de partida para el registro de la actividad de caza, tanto en las áreas situadas dentro como fuera de la RCTT. Observaciones directas fueron realizadas con la ayuda de cazadores locales 
que viven en la parte media del río Blanco. Tres cazadores, con sus respectivas familias, fueron capacitados durante 1993 para registrar la actividad de caza; de ellos, dos colaboraron de manera efectiva. Las viviendas de estos cazadores, llamados localmente como inspectores o registradores de caza, se encontraban estratégicamente ubicadas a la orilla del río Blanco a fin de visualizar y registrar con facilidad a los cazadores que iban de subida o de bajada. Durante los primeros seis meses del estudio, los registros de caza fueron controlados continuamente a fin de evaluar su funcionalidad y realizar los ajustes del caso. Posteriormente, éstos fueron controlados mensualmente y luego cotejados, a fin de complementar y uniformar la información entre uno y otro registrador. La colaboración de la mujer de los registradores de caza fue muy valiosa durante la ausencia del marido. Ellas continuaron registrando la actividad de caza cuando el marido iba de cacería o cuando llevaba a vender productos agropecuarios a la ciudad de Iquitos.

Cuando algunos cazadores no fueron controlados oportunamente, éstos fueron controlados indirectamente a través de la información proporcionada por habitantes del lugar o informantes clave. Estos informantes clave fueron aquéllos que testificaron haber observado in situ el número y sexo de los especímenes cazados y que no fueron reportados oportunamente al registrador de caza. Asimismo, estos informantes clave reportaron la venta de carne de monte del cazador no reportada en el mercado de Iquitos.

El registro de la caza incluía información sobre la cantidad cazada por especie, sexo, fechas de ingreso y salida del cazador. Esto último fue registrado para determinar los días de permanencia de caza.

En el caso de la RNPS, el registro de la caza se realizó con apoyo de cazadores locales pertenecientes a nueve comunidades, de modo similar a lo realizado en la RCTT.

\section{RESULTADOS}

\subsection{Fortalecimiento del sistema de co-manejo comunitario}

En la actualidad, el manejo de fauna silvestre con participación comunitaria representa una verdadera alternativa de conservación. Sin embargo, requiere de un proceso de co-manejo basado en las comunidades a fin de asegurar, en el largo plazo, el verdadero manejo comunal. Es decir, partir de iniciativas dadas y conducidas por las 
mismas comunidades, propiciando el aprovechamiento sostenible de aquellas especies aptas para ello y protegiendo aquellas especies cuya caza no está siendo realizada en los niveles sostenibles.

El manejo comunal se basa en la adquisición y comunicación de la información (Bodmer y Puertas, 2000). Para ello, se necesita utilizar el siguiente diseño: hacer que la gente local implemente el manejo de las poblaciones de caza. Los estudios biológicos de los animales de caza proveen información sobre el impacto de la caza y la efectividad de su manejo. Simultáneamente, la investigación y promoción agroforestal con énfasis en aguaje y chonta proveen información sobre el mejoramiento de hábitats de fauna silvestre y la efectividad de su manejo. Resultados de tales estudios son luego transmitidos a la gente local a través de actividades de promoción o extensión. Por cierto, el retorno de la información acerca de las poblaciones de animales de caza a la gente local, puede ser adecuadamente transmitida solo si contiene resultados de investigaciones complementarias y componentes de extensión. En otras palabras, el impacto del manejo puede ser determinado a través de investigaciones sobre las poblaciones de animales de caza que, luego, serán debidamente comunicadas a las comunidades mediante actividades de extensión. Por consiguiente, las investigaciones tienden el puente que liga las realidades de la biología poblacional con el manejo comunitario de los recursos naturales.

En el co-manejo deben participar, además de las comunidades locales, los siguientes actores: trabajadores de extensión o promotores de Organismos No Gubernamentales (ONG), funcionarios de Organismos Gubernamentales (OG) e investigadores (Bodmer y Puertas, 2000). Asimismo, se considera necesario considerar a los actores secundarios tales como comerciantes, localmente denominados "regatones", y habitantes de los principales poblados o ciudades.

El proceso de co-manejo basado en las comunidades, en el caso especifico del Tahuayo-Blanco, debe estar orientado expresamente al conocimiento de los niveles de caza, al análisis e interpretación de la caza, a la reglamentación y el cumplimiento de acuerdos sobre su manejo, en un ambiente armónico, de consenso comunal. A su vez, este proceso debe estar orientado a fortalecer la organización intra e intercomunitaria, a fin de monitorear de una manera efectiva el desarrollo de las actividades del manejo comunal participativo para conseguir los propósitos en el largo plazo.

Un paso importante en el co-manejo, con énfasis en el manejo comunal participativo de fauna silvestre, fue iniciado en Noviembre de 1993 en la zona del Tahuayo-Blanco y centralizada en la Comunidad El Chino. Acuerdos comunales para el cumpli- 
miento de un reglamento de caza, con la participación de moradores y autoridades políticas de las comunidades de Esperanza, Buena Vista, El Chino y otros habitantes del río Blanco fueron logrados. A través de las reuniones comunales y explicando a cada morador los alcances del reglamento, se promovió la participación de la gente local en un 80\%. Posteriormente, el 6 de marzo de 1994, en asamblea general con participación de dichas comunidades y principales actores, fue sustentado y aprobado dicho reglamento. Sin embargo, el reconocimiento legal a través de la firma del reglamento, dando amparo a los acuerdos comunales logrados, se obtuvo solo el 27 de marzo de 1994.

Durante 1995, factores adversos como la falta constante de los trabajadores de extensión, economía comunal debilitada por la creciente de los ríos Amazonas y Tahuayo, sistema tradicional de patronazgo imperante en la zona y corrupción de autoridades políticas y policiales locales, debilitaron el esquema de organización comunal. Ello debido a que tales personas vieron a la reserva como una oportunidad para satisfacer ambiciones personales a través de la extracción de carne de monte y madera, a pesar de sus restricciones legales. En consecuencia, el reglamento de caza quedó suspendido.

Sin embargo, un grupo compuesto por siete familias, pioneras de la creación de la reserva, decidió organizarse para combatir estratégicamente el sistema de corrupción imperante y continuar llevando a cabo el co-manejo basado en comunidades. La estrategia desarrollada fue: 1) la creación y el establecimiento permanente de una comunidad autónoma con el nombre de San Pedro, 2 ) la búsqueda de aliados en los antiguos habitantes del río Blanco, situados aguas arriba de San Pedro, mediante la creación de una comunidad anexa, con el mismo nombre de 7 de Julio y 3) denunciar a las autoridades competentes de las corrupciones habidas en El Chino. Esa estrategia dio los resultados esperados ya que hicieron caer el sistema de patronazgo y de corrupción de autoridades en la zona. A la fecha, aún es necesaria una coordinación intercomunitaria más efectiva.

En la actualidad, se sugiere que la Comunidad de San Pedro sea el centro de las actividades promotoras del co-manejo que coordine tanto con los moradores de las comunidades aledañas sobre el accionar de la reserva como también con los principales actores a nivel local. Sin embargo, para ello se requiere una efectiva presencia e integración de los extensionistas de fauna con los líderes de las comunidades beneficiarias. También se requiere que haya un constante flujo de información entre investigador y extensionista a fin de que este último sea un buen receptor y transmita adecuadamente la información recibida a los miembros de las comunidades encargadas del co-manejo. Experiencias habidas sugieren que una inadecuada transmisión 
de la información efectuada por los trabajadores de extensión puede hacer fracasar las acciones del co-manejo (Bodmer y Puertas, 2000). Desde luego, es necesaria una constante capacitación de los trabajadores de extensión, hecha por expertos en el manejo de fauna silvestre basado en comunidades. Por consiguiente, el principal propósito de este estudio es que en el largo plazo las propias comunidades puedan manejar la caza a través de la información obtenida por ellos mismos sobre el esfuerzo de caza.

Una vez fortalecido el sistema de manejo comunal a nivel local, el paso siguiente sería buscar el respaldo político a nivel regional, para así obtener el respaldo legal a los acuerdos y gestiones que las comunidades realizan en el contexto de manejo comunal. Para el logro de esto, las comunidades deben tener el respaldo de los principales actores a nivel local. Por otro lado, deben estar debidamente organizadas y capacitadas para llevar a cabo el co-manejo basado en comunidades.

\subsection{Importancia del manejo comunal para la conservación de las áreas protegidas}

Una manera promisoria para manejar la sobrecaza en las áreas protegidas, como la RNPS, sería tener áreas donde no se realice caza, o "áreas fuente", y las zonas adyacentes con caza persistente o "áreas sumidero", tanto en las zonas de uso como de amortiguamiento. El manejo de fauna silvestre debería tener un fuerte involucramiento comunal mediante un efectivo trabajo de extensión. El trabajo de extensión comunal debe tener en consideración los requerimientos biológicos de los animales de caza, así como de la realidad social y económica del poblador local. Recientemente, se ha dado mucha atención a las condiciones socio-económicas de la gente local y sus necesidades para llegar a tener una mejor condición de vida como un pre-requisito para el desarrollo sostenible (Pinkerton, 1989). Esto, sin duda, resalta la importancia de la conservación comunal en las áreas naturales protegidas. De igual importancia es el reconocimiento de que una mejor información biológica de las especies y ecosistemas debe incorporarse en los esfuerzos de la conservación comunal. Indudablemente, la conservación comunal fracasará si, atinadamente, no se toman en consideración ciertos atributos biológicos de las especies.

Como las comunidades locales dependen de la fauna silvestre para satisfacer sus necesidades básicas, el futuro de su conservación dependerá del uso sostenible de la misma. En ese sentido, estudios como el presentado en este artículo contribuirán al uso de las poblaciones de fauna silvestre de modo sostenible. 
Finalmente, tanto el uso sostenible como las estrategias de fuente-sumidero necesitan ser incluidas en las iniciativas de conservación comunal en las áreas naturales protegidas de la Amazonía con énfasis en la RNPS y la RCTT. Las comunidades locales de esas áreas necesitan reconocer el valor de las áreas sin caza como poblaciones fuente, ello a fin de garantizar el uso sostenible a largo plazo de sus recursos. Las comunidades que reconocen y consideran las áreas protegidas como zonas fuente lo hacen porque concuerdan en la necesidad de usar la fauna silvestre según sus realidad socio-económica. Por consiguiente, con áreas fuente o áreas protegidas funcionando como áreas fuente, la caza será sostenible y los esfuerzos del manejo comunal tendrán mas posibilidad de ser exitosos. Es decir, las comunidades tendrán mas interés en proteger las áreas debido al beneficio que obtendrían de un sistema de caza más sostenible. Por consiguiente, ello haría decrecer los conflictos entre los guardaparques y las comunidades locales y podría ser una alternativa financieramente sostenible para las áreas naturales protegidas.

\section{CONCLUSIONES}

Una alternativa viable para la conservación de los bosques amazónicos es hacer el manejo de los recursos naturales con la participación de las comunidades locales. Tanto la Reserva Nacional Pacaya Samiria como la Reserva Comunal TamshiyacuTahuayo representan excelentes modelos para esa alternativa. Sin embargo, para alcanzar los objetivos a largo plazo, el sistema de co-manejo requiere ser fortalecido, los hábitats de la fauna silvestre necesitan ser mejorados, la caza, pesca, recursos forestales y otros recursos naturales necesitan ser manejados por la gente local. Para alcanzar esta última meta, es muy importante que la gente local y las autoridades de gobierno tengan acceso a la información sobre el recurso a manejar.

La comunicación de la información necesita ser en un lenguaje con terminologías entendibles para promover su adecuado entendimiento y ampliar la participación en el análisis de uso del recurso a manejar. Por cierto, en el futuro la misma gente local analizaría la situación de sus recursos para luego decidir el manejo de la fauna silvestre mediante la implementación de un sistema participativo. 


\section{RECOMENDACIONES PARA LAS ACCIONES DE MANEJO COMUNAL}

Este estudio recomienda que la dirección para la conservación de la fauna silvestre, tanto en la RCTT como en la RNPS, debe tener en consideración los siguientes criterios: 1) tanto el uso sostenible como la estrategia fuente-sumidero necesitan ser incluidas en las iniciativas de manejo comunal de fauna silvestre en las áreas naturales protegidas de la amazonía, con énfasis en la RNPS y la RCTT; 2) es necesaria una adecuada actitud de las autoridades de gobierno, de los investigadores y trabajadores de extensión o promotores para proveer una adecuada información técnica y respetar las realidades socio-económicas de la gente local; 3) capacidad de una coordinación efectiva; 4) mucha energía y fuerza de voluntad por parte de las autoridades del gobierno para apoyar las gestiones del manejo comunal de fauna silvestre; 5) sentido común para entender tanto las necesidades de la gente local como las tendencias actuales de la cooperación técnica internacional y 6) alternativas económicas para la extracción no sostenible de la fauna silvestre.

El co-manejo debe ser realizado durante todo el proceso de realización de un plan de manejo de fauna silvestre, principalmente durante las fases de registro de información básica, diseño, implementación, ejecución y el monitoreo a dicho plan de manejo. Ello debido a que las comunidades nativas y ribereñas por sí solas no podrían conducir un plan de manejo ante un ecosistema complejo, con una débil economía y organización comunal, así como con una falta de respaldo gubernamental ante las iniciativas de manejo comunal.

Finalmente, a fin de hacer los programas de manejo de fauna silvestre de la RNPS y la RCTT más sostenibles, es necesario elaborar planes de manejo comprensivos que permitan cierta flexibilidad en las prácticas y generen un flujo de información en base a lecciones aprendidas. Eso dará lugar a la elaboración de programas adecuados a la situación local que, con apoyo del componente de extensión, recomiende a la gente local, ya sea cazadores o pescadores u otros manejadores, ajustar el uso de los recursos naturales según los límites sostenibles. Ello, según la característica biológica de las especies y las posibilidades de comercialización de los productos correspondientes. 


\section{AGRADECIMIENTOS}

Nuestro agradecimiento a los colegas y amigos que proporcionaron su valioso apoyo y sugerencias durante esta investigación, en especial a César Reyes, Etercit Pezo, James Penn, Rolando Aquino, David Freitas, Manuel Isuiza, José Mondragón, Sebastián Velarde y Grety Arriaga. También agradecemos al Instituto Nacional de Recursos Naturales (INRENA) y al Ministerio de Agricultura - Perú por las autorizaciones concedidas. Asimismo agradecemos al Centro de Investigaciones Veterinarias y de Altura (IVITA) de la Universidad Nacional Mayor de San Marcos (UNMSM) y a la University of Florida por las facilidades concedidas. Este trabajo fue financiado por Wildlife Conservation Society, the Chicago Zoological Society, Junglevagt for Amazonas (AIF-WWF/DK), Biodiversity Support Program (BSP), Rainforest Conservation Fund (RCF), Consejo Nacional de Ciencia y Tecnología (CONCYTEC) y la Asociación para la Conservación de la Amazonía (ACA). Estamos en deuda por el invalorable soporte proporcionado por las comunidades de la Reserva Nacional Pacaya Samiria y la Reserva Comunal Tamshiyacu-Tahuayo. Finalmente, nuestra gratitud a un recordado amigo y héroe de la conservación, Milton Chumbe Ayllón, con quien compartimos gratas experiencias de campo.

\section{BIBLIOGRAFIA}

ASOCIACIÓN PARA LA CONSERVACIÓN DE LA AMAZONIA (ACA). 1998. Community-based Natural Resources Management Programs in the Reserva Comunal Tamshiyacu-Tahuayo, Perú. Annual report. 45 pp.

ASPELIN, P. 1975. External articulation and domestic production: The artifact trade of the Maimainde of northwestern Mato Grosso, Brazil. In: Latin American Studies Program Dissertation, Series $N^{\circ}$ 59. Ithaca, N.Y.: Cornell University.

BECKERMAN, S. 1994. Hunting and fishing in Amazonia: Hold the Answers, What are the questions? In: A. Roosevelt (ed.). Amazonian Indians from Prehistory to the Present, Anthropological Perspectives. Tucson: University of Arizona Press. Pp. 177-200.

BODMER, R. 1993. Priorities for the conservation of mammals in the Peruvian Amazon. In: Oryx, 29(1): 23-28. 
1994. Managing wildlife with local communities: The case of the Reserva Comunal Tamshiyacu-Tahuayo. In: D. Western; M. Wright; S. Strum (ed.). Natural Connections: Perspectives on Community Based Management. Washington DC: Island Press. pp.113-134.

1995. Susceptibility of mammals to overhunting in Amazonia. In: J. Bissonette; P. Krausman (ed.). Integrating People and Wildlife for a Sustainable Future. Bethesda, Maryland: The Wildlife Society. pp. 292- 295.

BODMER, R.; FANG, T.; MOYA, L. 1988. Ungulate management and conservation in the Peruvian Amazon. In: Biological Conservation, 45: 303-310.

BODMER, R.; PENN, J.; FANG, T.; MOYA, L. 1990. Management programmes and protected areas. The case of the Reserva Comunal Tamshiyacu-Tahuayo, Peru. In: Parks, 1: 21-25.

BODMER, R.; PENN, J.; PUERTAS, P.; MOYA, L.; FANG, T. 1997. Linking conservation and local people through sustainable use of natural resources: Community-based management in the Peruvian Amazon. In: C. Freese (ed.). Harvesting Wild Species: Implications for Biodiversity Conservation. Baltimore, MD: The John Hopkins University Press. pp. 315-358.

BODMER, R.; PUERTAS, P. 2000. Community Based Co-Management of Wildlife in the Peruvian Amazon. In: J. Robinson; Bennet, L. (ed.). Hunting of Tropical Wildlife. University of Chicago Press.

BODMER, R.E.; PUERTAS, P. E.; GARCÍA, J.E.; DÍAS, D.; REYES, D. 1999. Game animals, palms and people of the flooded forests: Management considerations for the Pacaya-Samiria National Reserve. In: Ch. Padoch; J. Marcio Ayres; M. Pinedo-Vásquez; A. Henderson (ed.). Várzea Diversity, Development, and Conservation of Amazonia's Whitewater Floodplains. Advances in Economic Botany. New York: The New York Botanical Garden Press. Pp. 217-230.

COOMES, O. 1992. Making a Living in the Amazon Rainforest: Peasants, Land, and Economy in the Tahuayo River Basin of Northeastern Peru. Doctoral Dissertation. University of Wisconsin-Madison. 
DUFOUR, D. 1994. Diet and nutritional status of Amazonian peoples. In: A. Roosevelt (ed.). Amazonian Indians from Prehistory to the Present: Anthropological Perspectives. Tucson: University of Arizona Press. pp. 151-175.

EISENBERG, J. 1989. Mammals of the Neotropics: The Northern Neotropics, Vol. 1. Chicago: University of Chicago Press. 449 pp.

EMMONS, L.H. 1990. Neotropical Rainforest Mammals. A Field Guide. Chicago: University of Chicago Press. 281 pp.

FLOWERS, N.M. 1983. Seasonal Factors in subsistence, nutrition, and child growth in a central Brazilian Community. In: R.B. Hames; W.T. Vickers (ed.). Adaptive responses of Native Amazonians. New York: Academic Press. pp. 357-390.

1994. Subsistence strategy, social organization, and warfare in central Brazil in the context of European penetration. In: A. Roosevelt (ed.). Amazonian Indians from Prehistory to the Present: Anthropological Perspectives. Tucson: University of Arizona Press. pp. 249-269.

LITTLE, P.D. 1994. The link between local participation and improved conservation: A review of issues and experiences. In: D. Western; R.M. Wright; S.C. Strum (ed.). Natural Connections: Perspectives in Community-Based Conservation. Washington, DC: Island Press. pp.374-372.

MALTBY, E.; DUGAN, P.J.; LEFEUVRE, J.C. 1992. Conservation and development: The sustainable use of wetland resources. Gland, Switzerland: IUCN.

METCALFE, S. 1994. The Zimbabwe communal areas management program for Indigenous Resources (CAMPFIRE). In: D. Western; R. M. Wright (ed.). Natural Connections. Washington DC: Island Press. pp. 161-192.

NOWAK, R.; PARADISO, J. 1983. Walker's Mammals of the World, Vol. 1-2. $4^{\text {th }}$ edition. Baltimore: John Hopkins Press. 1362 pp.

Programa Integral de Desarrollo y Conservación Pacaya Samiria (PPS). 1995. Propuesta de Proyecto Segunda Fase. 25 pp. 
PENN, J.W. 1994. Agroforestería orientada a la fauna silvestre y necesidades humanas: desafios y realidades de la Reserva Comunal Tamshiyacu-Tahuayo. En: Kanatari, 490: 6-7; 491:6-7.

1999. The Aguaje Palm (Mauritia flexuosa L.f.): Examining its role as an Agroforestry Species in a Community Conservation Project. M.A. dissertation. USA: Universidad de Florida.

PINKERTON, E. 1989. Introduction: Attaining better fisheries management through co-management prospects, problems, and propositions. In: Pinkerton, E. (ed.). Co-operative Management of Local Fisheries: New Directions for Improved Management and Community Development. Vancouver: University of British Columbia Press. pp. 3-33.

POFFENBERGER, M. 1994. The resurgence of community forest management in Eastern India. In: D. Western; R.M. Wright; S.C. Strum (ed.). Natural Connections: Perspectives in Community-Based Conservation. Washington, DC: Island Press. pp.53-79.

PUERTAS, P.; BODMER, R. 1993. Conservation of a high diversity primate assemblage. In: Biodiversity and Conservation, 2: 586-593.

REDFORD, K.; ROBINSON, J. 1991. Subsistence and Commercial Uses of Wildlife in Latin America. In: J. Robinson; K. Redford (ed.). Neotropical Wildlife Use and Conservation. Chicago: University of Chicago Press. pp. 6-23.

ROBINSON, J.G.; BODMER, R. 1999. Invited paper: Towards wildlife management in tropical forests. In: Journal of Wildlife Management, 63 (1): 1-13.

ROBINSON, J.G.; REDFORD, K.H. 1991. Sustainable harvest of Neotropical forest animals. In: J.G. Robinson; K.H. Redford (ed.). Neotropical Wildlife Use and Conservation. Chicago: University of Chicago Press. pp. 415-429.

1994. Measuring the sustainability of hunting in tropical forests. In: Oryx, 25(4): 249-256.

TOWNSEND, W. 1996. Nyao Ito: Caza y Pesca de los Sirionó. Instituto de Ecología. Universidad Mayor de San Andrés FUND-ECO. 137 pp. 
VICKERS, W. 1980. An analysis of Amazonian hunting yields as a function of settlement age. In: R. Hames; K.M. Kesingers (ed.). Working Papers on South American Indians. Bennington: Bennington College, VT. pp. 7-29.

1991. Hunting yields and game composition over ten years in an Amazonian village. In: J. Ronbinson; K. Redford (ed.). Neotropical Wildlife Use and Conservation. Chicago: University of Chicago Press. pp. 53-81.

YOST, J.A.; KELLEY, P.M. 1983. Shotguns, blowguns, and spears: The analysis of technological efficiency. In: R.B. Hames; W.T. Vickers (ed.). Adaptive Responses of Native Amazonians. New York: Academic Press. pp. 189-224. 


\section{ANEXO}

Figura 1: Localización de las áreas de estudio en la Reserva Comunal TamshiyacuTahuayo y Reserva Nacional Pacaya Samiria. 1) área del Tahuayo-Blanco, 2) área del Samiria-Marañón y 3) área del Canal de Puinahua.

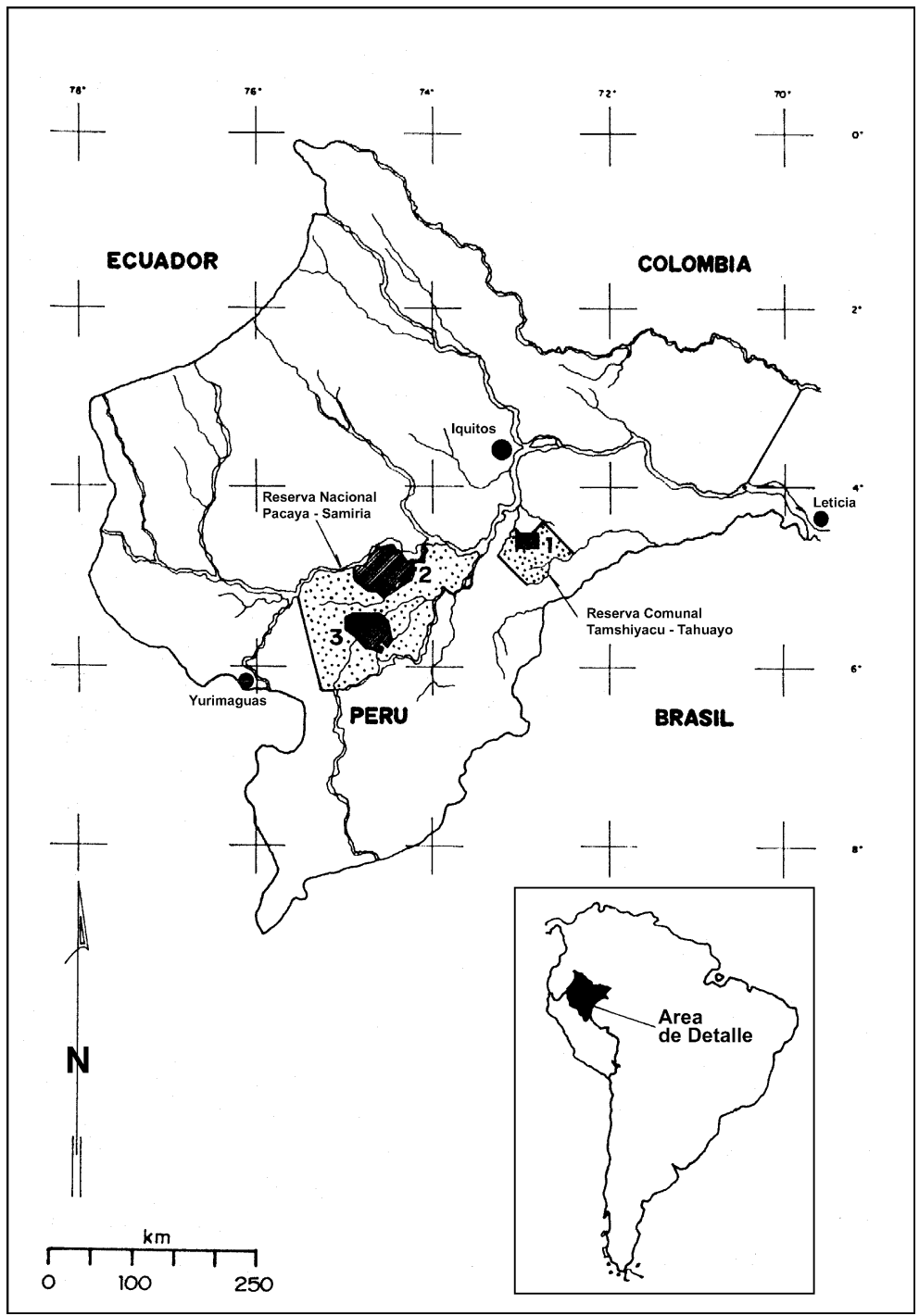

